\title{
Inclusive and differential vector boson (W, Z) measurements from CMS
}

\author{
Kadir Ocalan* on behalf of the CMS Collaboration \\ Necmettin Erbakan University, Konya, Turkey \\ E-mail: kadir.ocalan@cern.ch
}

\begin{abstract}
Weak vector boson $(\mathrm{W}, \mathrm{Z})$ production is one of the most prominent hard scattering processes at the LHC. Measurements of $\mathrm{W}$ and $\mathrm{Z}$ boson provide precision tests for the Standard Model including substantial inputs for parton distribution functions. The latest results on $\mathrm{W}$ and $\mathrm{Z}$ bosons and their productions in association with jets are presented based on proton-proton collision data recorded by the CMS detector at center-of-mass energies of $8 \mathrm{TeV}$ and $13 \mathrm{TeV}$. Precision measurements involving inclusive and differential production cross sections and their ratios for $\mathrm{W}$ and $\mathrm{Z}$ boson are reported as well as for $\mathrm{W}$ and $\mathrm{Z}$ boson produced in association with jets. The results are compared to predictions from various Monte Carlo event generators and theoretical calculations.
\end{abstract}

Corfu Summer Institute 2017 'School and Workshops on Elementary Particle Physics and Gravity' 2-28 September 2017

Corfu, Greece

\footnotetext{
* Speaker.
} 


\section{Introduction}

Measurements of standard model (SM) processes at hadron colliders involving weak vector boson production are crucial for probing the fundamental structure of the electroweak (EW) and the strong interactions. Clean experimental signatures and large production cross sections of $\mathrm{W}$ and $\mathrm{Z}$ bosons enable precision tests of SM and provide substantial inputs for parton distribution functions (PDFs), Monte Carlo (MC) event generators, and higher order theoretical calculations. W/Z boson measurements are important for improving background modeling for rare SM processes and beyond SM signatures. W/Z boson is reconstructed using leptonic final states $\left(Z / \gamma^{*} \rightarrow l^{+} l^{-}, W^{ \pm} \rightarrow l^{ \pm} v\right.$, where $l$ is an electron (e) and/or a muon $(\mu)$ and $v$ is detector escaping neutrino identified by significant amount of missing energy) either with or without presence of accompanying hadronic jets and thus their measurements are used for detector calibration exploiting lepton, missing energy, and jet information. Their productions are characterized by measuring inclusive and differential cross sections and also ratios of cross sections with higher precision. In this report, the latest results on W/Z boson production and also on their production in association with jets are presented from proton-proton (pp) collision data collected at center-of-mass energies of $8 \mathrm{TeV}$ and $13 \mathrm{TeV}$ by the Compact Muon Solenoid (CMS) experiment [1] at the CERN Large Hadron Collider (LHC).

\section{Inclusive and differential $\mathrm{W} / \mathrm{Z}$ boson production measurements}

Total and fiducial inclusive $\mathrm{W}(l v)$ and $\mathrm{Z}(l l)$ boson cross sections and their ratios are measured using a data sample at $13 \mathrm{TeV}$ corresponding to an integrated luminosity of $43 \mathrm{pb}^{-1}$ recorded by the CMS detector [2]. Events are selected with lepton transverse momentum $p_{T}(l)>25 \mathrm{GeV}$ and pseudorapidity $|\eta(e)|(|\eta(\mu)|)<2.5$ (2.4) requirements and $\mathrm{Z}$ boson candidates are required to be in the dilepton invariant mass window of $60-120 \mathrm{GeV}$. The measured inclusive cross sections are compared with next-to-next-to-leading-order (NNLO) prediction by FEWZ. The measured inclusive cross sections and ratios of cross sections agree with NNLO SM prediction using NNPDF 3.0 PDF set as summarized in Figure 1.

$\mathrm{Z}(\mu \mu)$ boson production cross section is measured both inclusively and differentially using a data sample of $2.3 \mathrm{fb}^{-1}$ at $13 \mathrm{TeV}$ [3]. Events are selected with $p_{T}(\mu)>25 \mathrm{GeV}$ and $(|\eta(\mu)|)<$ 2.4 requirements in the dimuon invariant mass range of $60-120 \mathrm{GeV}$. The measured total inclusive cross section times branching fraction $\mathscr{B}$ is $\sigma(\mathrm{pp} \rightarrow \mathrm{Z}+\mathrm{X}) \mathrm{x} \mathscr{B}\left(\mathrm{Z} \rightarrow \mu^{+} \mu^{-}\right)=1870 \pm 2$ (stat.) \pm 35 (syst.) \pm 51 (lumi.) pb and consistent with NNLO quantum chromodynamics (QCD) calculation of $1870 \pm 50 \mathrm{pb}$ by FEWZ using NNPDF 3.0 PDF set, where next-to-leading-order (NLO) EW radiative corrections are included. Differential $\mathrm{Z}(\mu \mu)$ production cross section is measured as a function of $\phi_{\eta}^{*}$ variable [4] which is a probe of strong interaction and correlated to $p_{T}(Z)$ having relatively smaller experimental uncertainty. The unfolded data for the differential $\phi_{\eta}^{*}$ measurement is in fair agreement with the predictions by MadGraph5_aMC@NLO with NLO matrix elements, POWHEG at NLO, and FEWZ at NNLO as shown in Figure 2, except for the observed discrepancy in the low- $\phi_{\eta}^{*}$ region by FEWZ due to lack of soft gluon resummation.

$\mathrm{W}(l v)$ and $\mathrm{Z}(\mu \mu)$ boson production cross sections and their ratios $\left(\mathrm{W}^{-}\right.$to $\mathrm{W}^{+}$and $\mathrm{Z}$ to $\left.\mathrm{W}\right)$ are measured differentially using a lower luminosity data sample of $18.4 \mathrm{pb}^{-1}$ at $8 \mathrm{TeV}$ with phase 


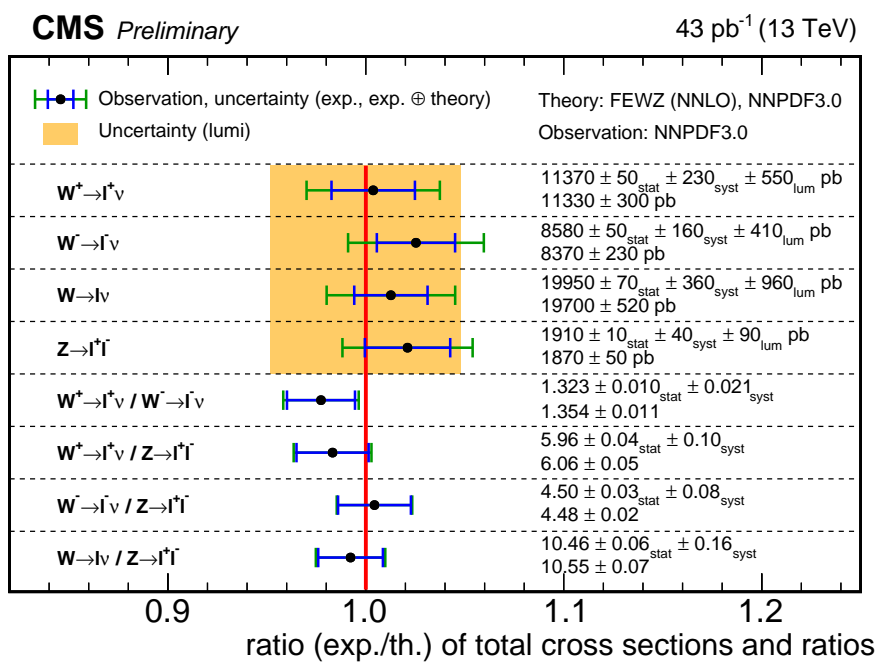

Figure 1: Measured total inclusive $\mathrm{W}^{+}, \mathrm{W}^{-}, \mathrm{W}$, and $\mathrm{Z}$ boson production cross sections times branching fractions, $\mathrm{W}$ to $\mathrm{Z}$ and $\mathrm{W}^{+}$to $\mathrm{W}^{-}$ratios, and their theoretical predictions [2]. The uncertainty due to the luminosity measurement is indicated by the shaded box. The inner error bars correspond to the experimental uncertainties, while outer error bars also include the uncertainties in the theoretical predictions. The measured and predicted cross sections are given numerically on the right.
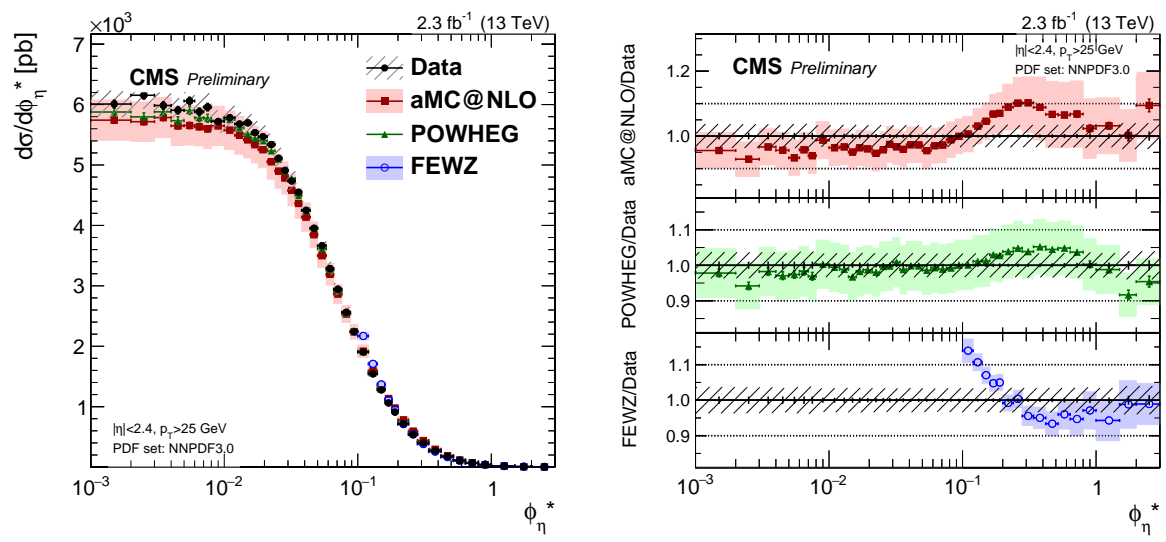

Figure 2: Differential cross section measurement of $\mathrm{Z}(\mu \mu)$ boson for the $\phi_{\eta}^{*}$ variable (left) with predictionto-data cross section ratios (right) [3]. The data points (black) are surrounded by the total uncertainty (shaded band) and its statistical component (solid line). The data is compared with MadGraph5_aMC@NLO (square red markers), POWHEG (green triangles), and FEWZ (empty blue circles) predicitons. The solid band around the predictions represents the total uncertainty, while the solid line corresponds to the statistical component. 
space requirements of $p_{T}(e, \mu)>25,20 \mathrm{GeV}$ and $|\eta(e, \mu)|<2.5,2.1$ in respective order of the decay modes [5]. The results are compared with the theoretical predictions by ResBos, POWHEG, and FEWZ using CT10 PDF set. The predictions are able to describe the data, except for the expected deviation by FEWZ at low- $p_{T}(Z)$ region as shown in the prediction-to-data comparisons in Figure 3.
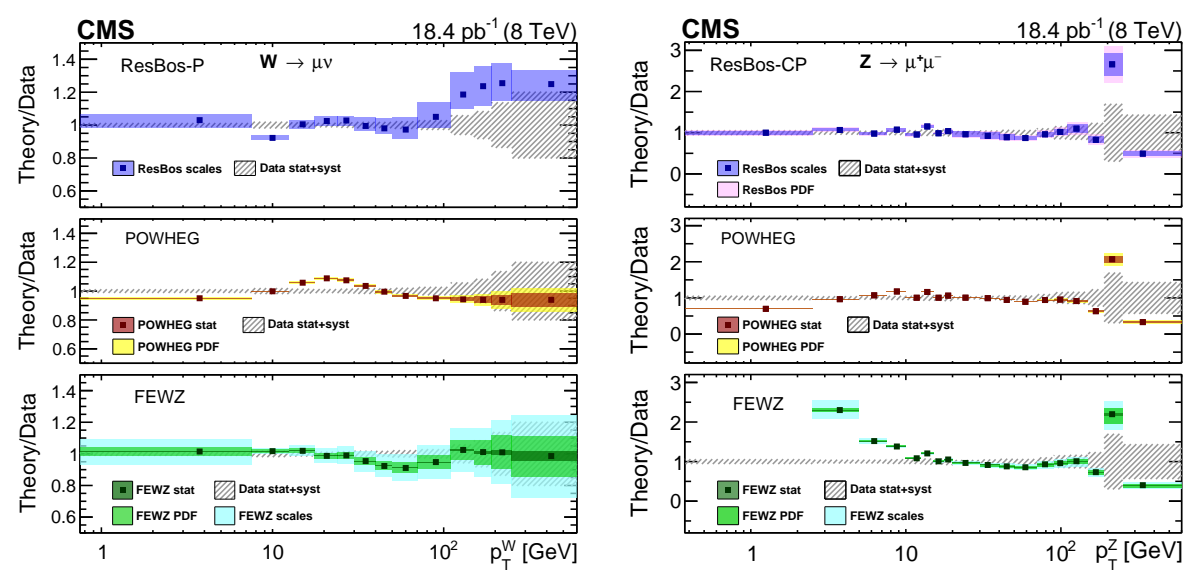

Figure 3: Theory prediction-to-data ratios of differential cross section measurements as functions of $p_{T}(W)$ (left) and $p_{T}(Z)$ (right) for $\mu$ decay channels at $8 \mathrm{TeV}$ [5]. The bands include the statistical uncertainties, uncertainties from scales, and PDF uncertainties for FEWZ; the statistical uncertainties and PDF uncertainties for POWHEG; the uncertainty from scales for ResBos-P; the uncertainty from scale and PDF variation for ResBos-CP; and the sum of the statistical and systematic uncertainties in quadrature for data.

The differential cross section and charge asymmetry for inclusive $\mathrm{W}^{ \pm}\left(\mu^{ \pm} v\right)$ boson production are measured using $18.8 \mathrm{fb}^{-1}$ data at $8 \mathrm{TeV}$ based on a muon selection of $p_{T}(\mu)>25 \mathrm{GeV}$ and $|\eta(\mu)|<2.5$ [6]. The measured differential cross section and $\mathrm{W}$ boson charge asymmetry as a function of $|\eta(\mu)|$ are well described by FEWZ prediction at NNLO calculated using different PDF sets. The results of the charge asymmetry measurement are incorporated into a QCD analysis at NNLO in combination with deep inelastic electron(positron)-proton scattering (DIS) data at HERA [8] and provide significant constraints on the ratio of $u$ and $d$ valence quark distributions in the proton for values of $\mathrm{x}$, the Bjorken scaling variable [7], between $10^{-3}$ and $10^{-1}$ as shown in Figure 4.

\section{Drell-Yan production measurements}

Drell-Yan lepton pairs are produced via $\mathrm{Z} / \gamma^{*}$ exchange in the s-channel at hadron colliders. The differential and double differential Drell-Yan cross sections are measured in the dielectron and dimuon channels based on a data sample corresponding to $19.7 \mathrm{fb}^{-1}$ at $8 \mathrm{TeV}$ [9]. In a more recent measurement, differential cross section of Drell-Yan lepton pairs is measured using $2.8 \mathrm{fb}^{-1}$ data at $13 \mathrm{TeV}$ in the dimuon invariant mass range of $15-3000 \mathrm{GeV}$ [10]. The measured differential cross section is in agreement with the MadGraph5_aMC@NLO and FEWZ 3.1 NNLO predictions using NNPDF 3.0 PDF set as shown in Figure 5 (left) as a function of the dilepton invariant mass. Measurements of Drell-Yan production in terms of mass and rapidity distributions are sensitive to 

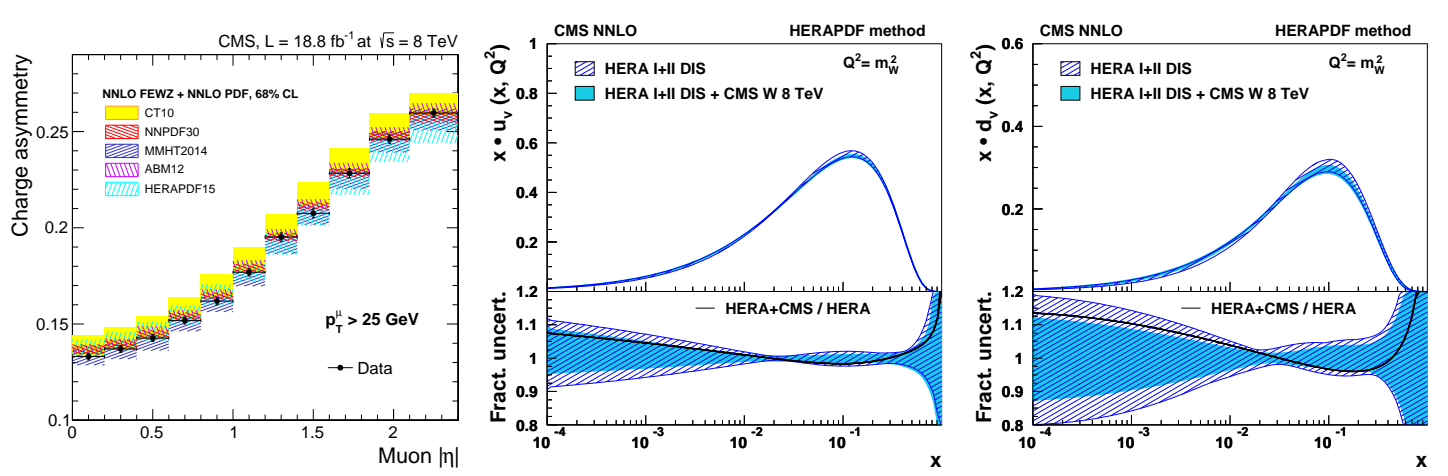

Figure 4: Comparison of $\mathrm{W}$ boson charge asymmetries to NNLO predictions calculated using the FEWZ 3.1 MC tool interfaced with different PDF sets (left) [6]. The smaller vertical error bars on the data points include the statistical and systematic uncertainties, while the full error bars include the integrated luminosity uncertainty. The PDF uncertainty of each PDF set is shown by a shaded (or hatched) band. Distributions of $\mathrm{u}$ valence (middle) and d valence (right) quarks as a function of scaling variable $\mathrm{x}$ [6]. The results of the fit to the HERA data and muon asymmetry measurements (light shaded band), and to HERA data only (hatched band) are compared.

parton content of the proton. The differential cross section is also measured for Drell-Yan events in both dielectron and dimuon channels as a function of $\phi^{*}$ variable at $8 \mathrm{TeV}$ [11]. None of the theoretical calculations predict the measurement perfectly over the entire range of $\phi^{*}$ as shown in Figure 5 (right).

The forward-backward asymmetry of oppositely charged lepton pairs produced via $\mathrm{Z} / \gamma^{*}$ boson exchange is measured using a data sample of $19.7 \mathrm{fb}^{-1}$ at $8 \mathrm{TeV}$ [12]. The measurement is performed for dilepton invariant mass window of $40-2000 \mathrm{GeV}$ and for dilepton rapidity up to 5. The measured forward-backward asymmetries are consistent with the SM prediction by POWHEG NLO using CT10 PDFs. Measurements of forward-backward asymmetry is sensitive to new physics, quark weak couplings, and effective weak mixing angle. The effective weak mixing angle using the forward-backward asymmetry of Drell-Yan events is measured at $8 \mathrm{TeV}$ [13]. The effective weak mixing angle is extracted from the combined lepton data samples using template fitting in bins of dilepton invariant mass and rapidity as $\sin ^{2} \theta_{\text {lept }}^{\text {eff }}=0.23101 \pm 0.00036$ (stat.) \pm 0.00018 (syst.) \pm 0.00016 (theory) \pm 0.00030 (pdf). The results are compared to the previous measurements from different experiments and are consistent with the most precise LEP and SLD measurements [14] as shown in Figure 6.

\section{4. $W / Z$ boson production in association with jets}

W/Z boson production in association with jets (W/Z+jets) processes are important SM benchmarks that provide precision tests for perturbative QCD (pQCD) calculations and valuable inputs to constrain PDFs. Typical fiducial phase space selections for W/Z+jets production are $p_{T}(l)>20$ or $25 \mathrm{GeV},|\eta(l)|<2.4$, dilepton mass range of $71-111 \mathrm{GeV}$ for $\mathrm{Z}$ boson production, W boson transverse mass $m_{T}>50 \mathrm{GeV}$ for $\mathrm{W}$ boson production, and anti- $\mathrm{k}_{T}$ [15] jets with $p_{T}($ jet $)>30 \mathrm{GeV}$ and rapidity $|y(j e t)|<2.4$. Differential cross sections for $\mathrm{W}(\mu v)+$ jets up to 7 jets for the jet multiplicity and 4 inclusive jets for the jet kinematical and angular correlation variables are measured 

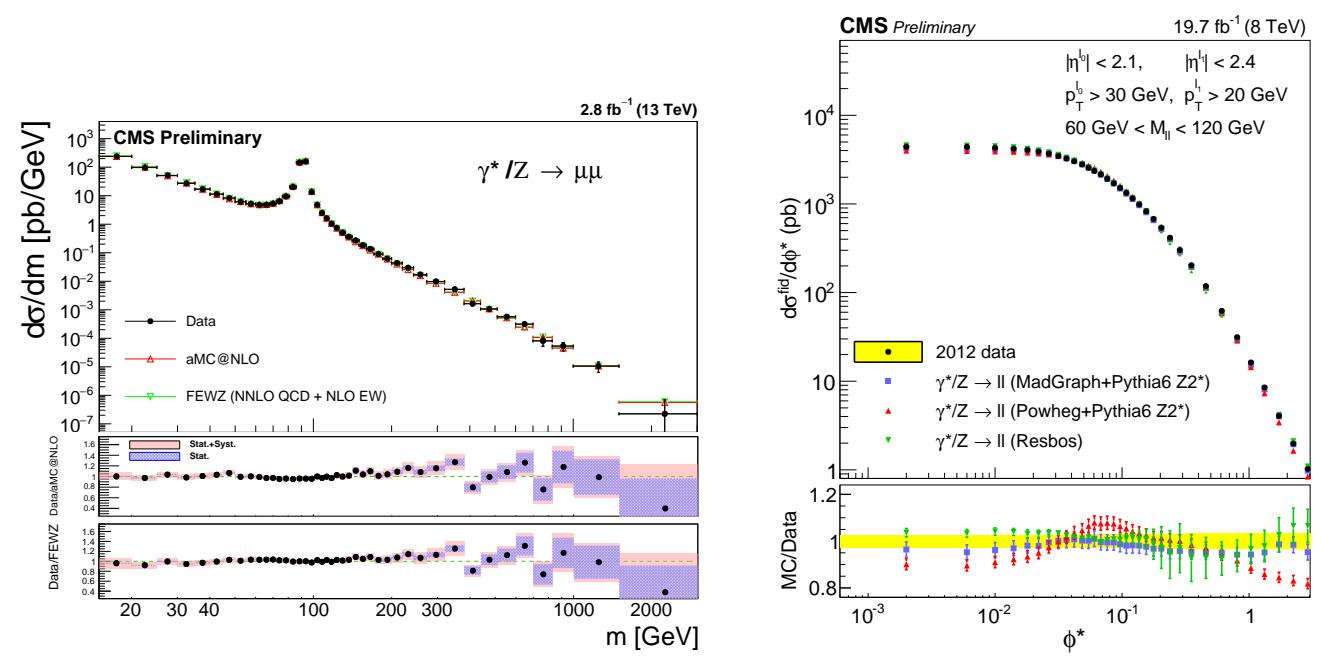

Figure 5: The Drell-Yan differential cross section measurement at $13 \mathrm{TeV}$ as a function of dimuon invariant mass compared to the predictions (left) [10]. The band with red color denotes total uncertainty which is the combination of statistical, systematical, theoretical, and luminosity uncertainties, while the band with purple color denotes the statistical uncertainty only. The differential cross section measurement for DrellYan events in the combined lepton channel as a function of $\phi^{*}$ variable at $8 \mathrm{TeV}$ compared to the predictions (right) [11]. The horizontal band corresponds to the experimental uncertainty. The vertical bars correspond to the statistical, scale and PDF uncertainties in the theoretical predictions.

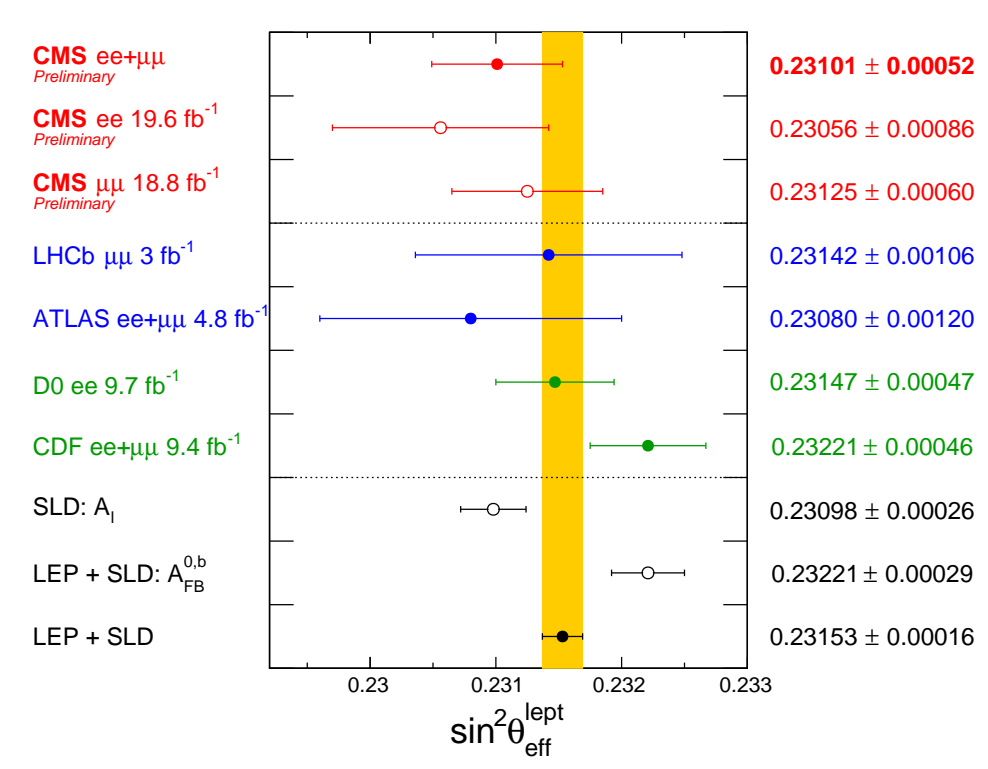

Figure 6: Comparison of the measured $\sin ^{2} \theta_{\text {lept }}^{\text {eff }}$ in the dimuon and dielectron channels and their combination with the previous LEP, SLC, Tevatron and LHC measurements [13]. The shaded band corresponds to the combination of the LEP and SLC measurements. 
at $8 \mathrm{TeV}$ [16]. The measured differential cross sections are compared with recent event generators, as well as NLO and NNLO theoretical predictions. The measured range for the leading jet $p_{T}$ extends up to $1 \mathrm{TeV}$ and the measurement and the predictions are in generally good agreement within uncertainties over the entire range as shown in Figure 7 (left). The first differential cross section measurement for $\mathrm{W}(\mu v)+\mathrm{jets}$ at $13 \mathrm{TeV}$ is performed by the CMS Collaboration based on a data sample of $2.2 \mathrm{fb}^{-1}$ [17]. The leading jet $p_{T}$ distribution is well reproduced by the predictions at leading-order (LO), NLO, and NNLO as shown in Figure 7 (right). The cross section is also measured at $13 \mathrm{TeV}$ differential in angular distance variable calculated between $\mu$ and the closest jet $\Delta \mathrm{R}(\mu$, closest jet $)$ which probes contribution of electroweak radiative processes to $\mathrm{W}+\mathrm{jets}$ events. The NNLO calculation gives the best agreement with the data for $\Delta \mathrm{R}(\mu$, closest jet $)$ as shown in Figure 8 (left). The differential cross sections are measured also for $\mathrm{Z}+\mathrm{jets}$ events in both dielectron and dimuon channels at $8 \mathrm{TeV}$ as functions of numerous observables up to 5 inclusive jets [18]. The data description is improved by the predictions when NLO terms are included as shown for the leading jet $p_{T}$ in Figure 8 (right).
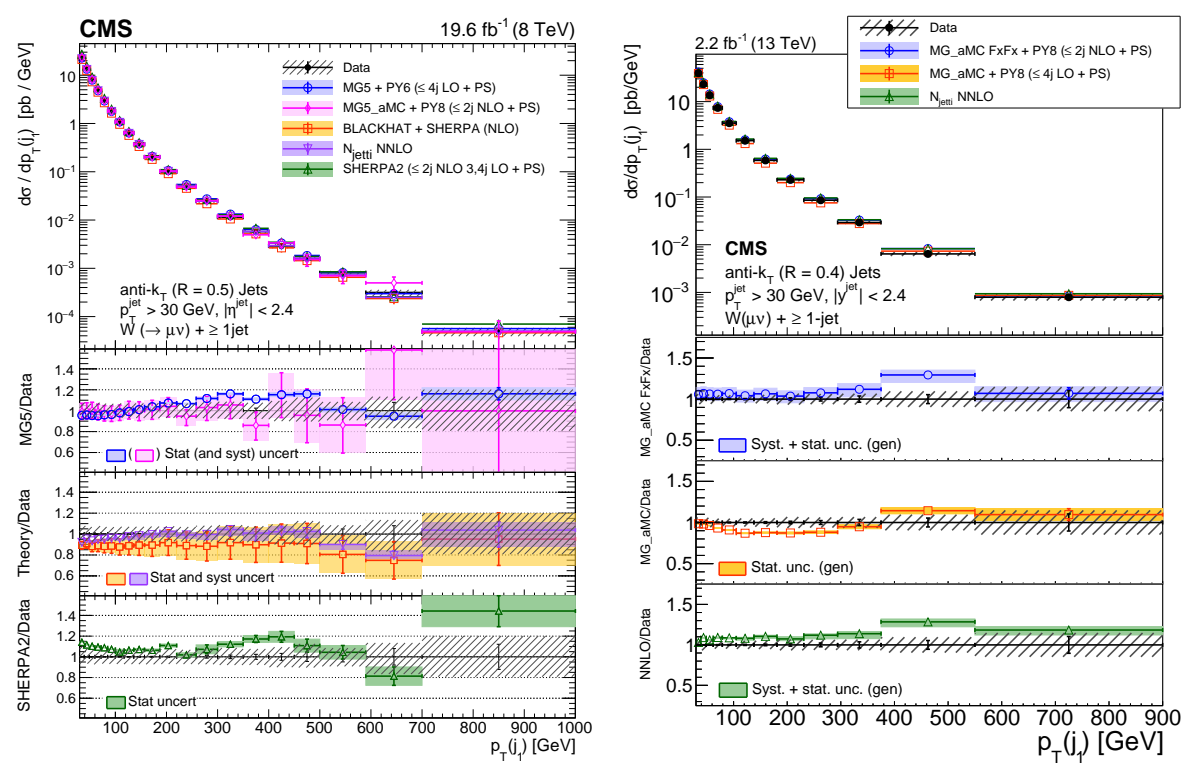

Figure 7: The differential cross section for $\mathrm{W}(\mu v)+$ jets production as a function of the leading jet $p_{T}$ at $8 \mathrm{TeV}$ [16] (left). The measurement is compared with the predictions at different orders in pQCD. Black circular markers with gray hatched band represent unfolded data measurements and their total uncertainties. Overlaid are the predictions together with their uncertainties. The differential cross section for $\mathrm{W}(\mu v)+\mathrm{jets}$ production as a function of the leading jet $p_{T}$ at $13 \mathrm{TeV}$ [17] (right). The MadGraph5_aMC@NLO prediction is given only with its statistical uncertainty. The bands around the MadGraph5_aMC@NLO FxFx and NNLO predictions represent their theoretical uncertainties including both statistical and systematic components. The lower panel shows the ratios of the prediction to the unfolded data.

W/Z boson production processes in association with heavy flavor jets (W/Z+HF-jets) have been measured in the CMS experiment. W/Z+HF-jets analyses are experimentally challenging but they are of paramount importance as they provide precision tests for QCD HF sector and are sensitive to probe HF quark (bottom (b) and charm (c) quarks) content in the proton. In the CMS experiment, HF jets are identified using multivariate analysis techniques (information about sec- 

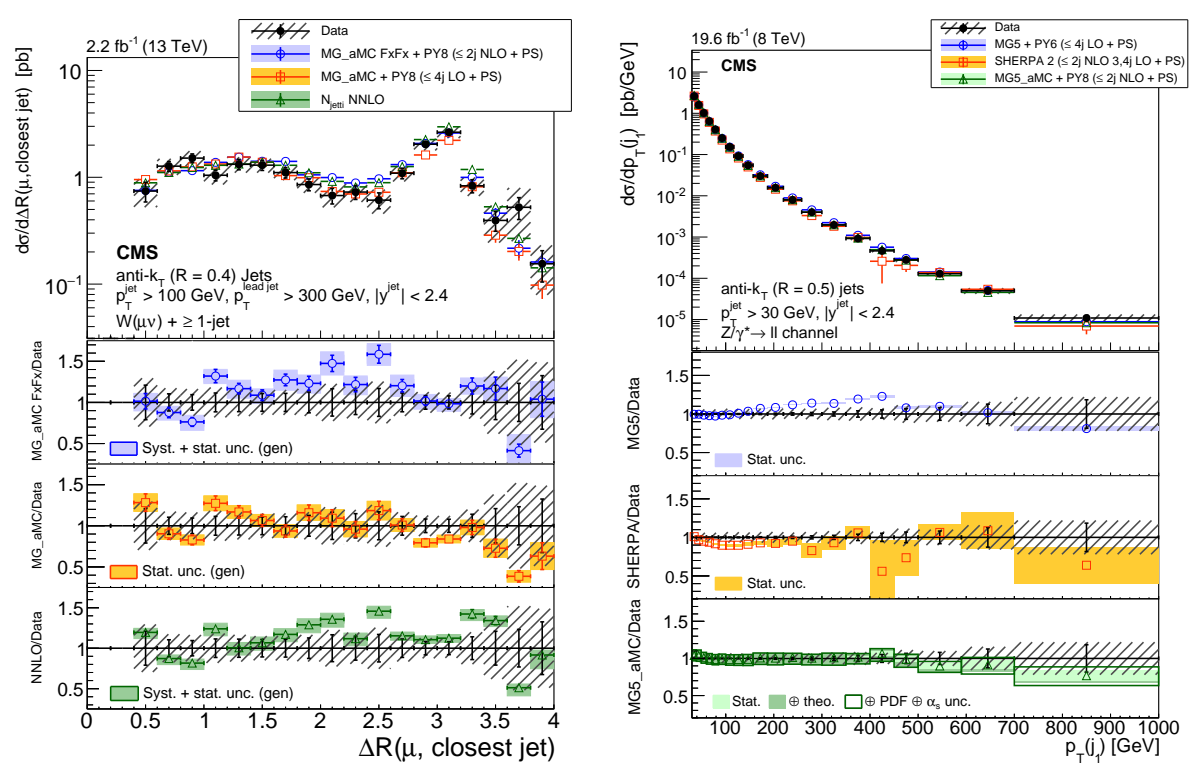

Figure 8: The differential cross section measurement for $\mathrm{W}(\mu v)+\mathrm{jets}$ events as a function of the $\Delta \mathrm{R}(\mu$, closest jet) variable at $13 \mathrm{TeV}$, compared to the predictions of MadGraph5_aMC@NLO FxFx, MadGraph5_aMC@NLO at LO, and the NNLO calculation [17] (left). The differential cross section for $\mathrm{Z}(l l)+$ jets at $8 \mathrm{TeV}$ as a function of the first leading jet $p_{T}$ [18] (right). Error bars around the experimental points show the statistical uncertainty, while the cross-hatched bands indicate the total experimental uncertainty. The boxes around the MadGraph5_aMC@NLO to measurement ratio represent the uncertainty on the prediction, including statistical, theoretical (from scale variations), and PDF uncertainties.

ondary vertices and impact parameters) or explicitly requiring $b$ and $c$ hadrons or soft muons in jets. The differential cross sections for the associated production of a $\mathrm{Z}(l l)$ boson and at least one $\mathrm{b}$ jet and two $\mathrm{b}$ jets as well as the ratios of differential cross sections for the associated production with at least one $b$ jet to the associated production with any jet are measured at $8 \mathrm{TeV}$ [19]. The results are compared to the theoretical predictions, testing two different flavor schemes (FS) for the choice of initial-state partons as shown for the leading $\mathrm{b}$ jet $p_{T}$ in Figure 9 (left). The predictions show discrepancy at low leading $\mathrm{b}$ jet $p_{T}$ region for $\mathrm{Z}(l l)+\geq 1-\mathrm{b}$ jet and the measurements do not strongly discriminate between 4FS and 5FS approaches used in the event generation. The associated production of a $\mathrm{Z}(l l)$ boson and at least one $\mathrm{c}$ jet is studied at $8 \mathrm{TeV}$ [20]. The relative production of a $\mathrm{Z}$ boson and at least one $\mathrm{c}$ or $\mathrm{b}$ jet is measured in terms of cross sections ratio as $\sigma(\mathrm{pp} \rightarrow \mathrm{Z}+\mathrm{c}+\mathrm{X}) / \sigma(\mathrm{pp} \rightarrow \mathrm{Z}+\mathrm{b}+\mathrm{X})=2.0 \pm 0.2$ (stat.) \pm 0.2 (syst.). The $\mathrm{Z}+\mathrm{c}$ production cross section and the cross sections ratio are also measured differentially as a function of $p_{T}(Z)$ and compared with several theoretical predictions. TheMadGraph5_aMC@NLO predictions describe well the measurement for $Z(l l)+\geq 1$-c jet events as shown in Figure 9 (right).

\section{EW W/Z+2-jets production measurements}

EW production of $\mathrm{W} / \mathrm{Z}+2$-jets processes are characterized by the presence of two high- $p_{T}$ jets with large $\eta$ separation and low hadronic activity in-between. Measurements of these processes provide tests of the EW sector (i.e. gauge boson self interactions) and are important for Higgs boson 

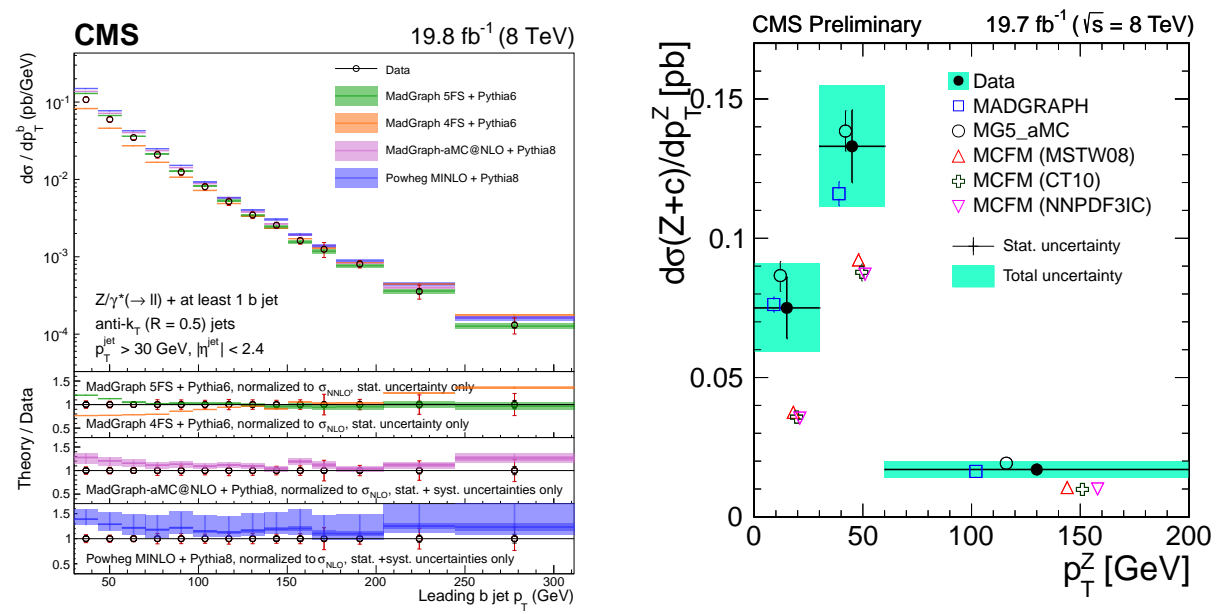

Figure 9: The differential cross section measurement for $\mathrm{Z}(l l)+\geq 1$-b jet production as a function of the leading $\mathrm{b}$ jet $p_{T}$, compared with the theoretical predictions [19] (left). For each data point the statistical and the total uncertainties are represented by the double error bar. The differential cross section measurement for $\mathrm{Z}(l l)+\geq 1$-c jet production as a function of the $p_{T}(Z)$, compared with the theoretical predictions [20] (right). Statistical and systematic uncertainties in the theoretical predictions are shown added in quadrature.

studies in vector boson fusion processes. Cross section measurement of the $\mathrm{EW} \mathrm{W}(l v)+2$-jets final state is measured at $8 \mathrm{TeV}[21]$ in the kinematic region defined as $p_{T}\left(j_{1}\right)>60 \mathrm{GeV}, p_{T}\left(j_{2}\right)>50$ $\mathrm{GeV}$, and $|\eta(j)|<4.7$ with high dijet invariant mass $m_{j j}>1000 \mathrm{GeV}$ and small hadronic activity in the central region of the detector. This is the first cross section measurement for this process in which the fiducial cross is measured as $\sigma(\mathrm{EW} \mathrm{W}(l v)+2$-jets $)=0.42 \pm 0.04$ (stat.) \pm 0.09 (syst.) \pm 0.01 (lumi.) $\mathrm{pb}$ and in agreement with the SM LO prediction of $\sigma(\mathrm{SM} \mathrm{LO} \mathrm{EW} \mathrm{W}(l v)+2$-jets $)=$ $0.50 \pm 0.02$ (scale) \pm 0.02 (PDF) pb by MadGraph5_aMC@NLO. In a more recent measurement at $13 \mathrm{TeV}$ using a data sample of $35.9 \mathrm{fb}^{-1}$, cross section for the pure EW Z $(l l)+2$-jets final state is measured [22] in the kinematic region defined as $p_{T}(j)>25 \mathrm{GeV}, m_{j j}>120 \mathrm{GeV}$, and dilepton invariant mass $\mathrm{m}_{l l}>50 \mathrm{GeV}$. The measured fiducial cross section $\sigma(\mathrm{EW} \mathrm{Z}(l l)+2$-jets $)=552 \pm 19$ (stat.) \pm 55 (syst.) fb is in agreement with the SM LO prediction $\sigma$ (SM LO EW Z $(l l)+2$-jets $)=$ $543 \pm 24$ fb by MadGraph5_aMC@NLO event generator.

\section{Conclusion}

The CMS Collaboration has provided a comprehensive set of measurements on W/Z (+jets) production from 8 and $13 \mathrm{TeV}$ pp collision data at the LHC. High precision has been achieved in the measurements of inclusive and differential cross sections and ratios of cross sections using new experimental methods and large datasets. The tested predictions comprising matrix element calculations, parton shower models, and (N)NLO fixed order calculations are generally in good agreement with the measurements. The presented measurements provided a broad range of tests for the QCD and EW sector with wealth of information in terms of perturbative and soft QCD effects, PDF constraints, Drell-Yan forward-backward asymmetry, W charge asymmetry, weak mixing angle $\sin ^{2} \theta_{\text {lept }}^{\text {eff }}$, background modeling for Higgs boson and new physics processes, HF sector, and gauge boson self interactions. There is still need to improve modeling and precisions 
for the remained discrepancies and to constrain experimental uncertainties using larger datasets with the ongoing and near future CMS W/Z (+jets) measurements.

\section{Acknowledgments}

The author acknowledges the financial support for the workshop by Necmettin Erbakan University Scientific Research Projects Unit (BAP) under the AGED1 project with number 172518001703.

\section{References}

[1] The CMS Collaboration, The CMS Experiment at the CERN LHC, JINST 3 (2008) S08004.

[2] The CMS Collaboration, Measurement of inclusive $W$ and $Z$ boson production cross sections in pp collisions at $13 \mathrm{TeV}, \mathrm{CMS}-\mathrm{PAS}-\mathrm{SMP}-15-004, \mathrm{http} / / / \mathrm{cds} . c e r n . c h /$ record/2093537.

[3] The CMS Collaboration, Measurements of inclusive and differential Z boson production cross sections in pp collisions at $13 \mathrm{TeV}$, CMS-PAS-SMP-15-011, http://cds.cern.ch/record/2140105.

[4] A. Banfi, M. Dasgupta, S. Marzani, and L. Tomlinson, Probing the low transverse momentum domain of Z production with novel variables, JHEP 01 (2012) 044 [arXiv: 1110 . 400 9].

[5] The CMS Collaboration, Measurement of the transverse momentum spectra of weak vector bosons produced in proton-proton collisions at 8 TeV, JHEP 02 (2017) 096 [arXiv: 1606.05864 ].

[6] The CMS Collaboration, Measurement of the differential cross section and charge asymmetry for inclusive pp $\rightarrow W^{ \pm}+X$ production at 8 TeV, Eur. Phys. J. C 76 (2016) 469 [arXiv: 1603.01803 ].

[7] J. D. Bjorken and E. A. Paschos, Inelastic electron-proton and $\gamma$-proton scattering and the structure of the nucleon, Phys. Rev. 01 (1969) 1975.

[8] The H1 and ZEUS Collaborations, Combination of measurements of inclusive deep inelastic ep scattering cross sections and QCD analysis of HERA data, Eur. Phys. J. C 75 (2015) 580 [arXiv:1506.06042].

[9] The CMS Collaboration, Measurements of differential and double-differential Drell-Yan cross sections in proton-proton collisions at 8 TeV, Eur. Phys. J. C 75 (2015) 147 [arXiv: 1412.1115 ].

[10] The CMS Collaboration, Measurement of the differential Drell-Yan cross section in proton-proton collisions at $13 \mathrm{TeV}$, CMS-PAS-SMP-16-009, http://cds.cern.ch/record/2205152.

[11] The CMS Collaboration, Measurements of $\phi^{*}$ differential cross sections for Drell-Yan events in pp collisions at $8 \mathrm{TeV}$, CMS-PAS-SMP-15-002, http://cds.cern.ch/record/2194259.

[12] The CMS Collaboration, Forward-backward asymmetry of Drell-Yan lepton pairs in pp collisions at 8 TeV, Eur. Phys. J. C 76 (2016) 325 [arXiv: 1601.04768$].$

[13] The CMS Collaboration, Measurement of the weak mixing angle with the forward-backward asymmetry of Drell-Yan events at $8 \mathrm{TeV}$, CMS-PAS-SMP-16-007, https://cds.cern.ch/record/2273392.

[14] The ALEPH Collaboration, The DELPHI Collaboration, The L3 Collaboration, The OPAL Collaboration, The SLD Collaboration, The LEP Electroweak Working Group, The SLD Electroweak and Heavy Flavour Groups, Precision electroweak measurements on the Z resonance, Phys. Rept. 427 (2006) 257 [arXiv: hep-ex/0509008]. 
[15] M. Cacciari, G. P. Salam, and G. Soyez, The anti-kt jet clustering algorithm, JHEP 04 (2008) 063 [arXiv:0802.1189].

[16] The CMS Collaboration, Measurements of differential cross sections for associated production of a W boson and jets in proton-proton collisions at 8 TeV, Phys. Rev. D 95 (2017) 052002 [arXiv:1610.04222].

[17] The CMS Collaboration, Measurement of the differential cross sections for the associated production of a W boson and jets in proton-proton collisions at 13 TeV, Phys. Rev. D 96 (2017) 072005 [arXiv:1707.05979].

[18] The CMS Collaboration, Measurements of differential production cross sections for a $Z$ boson in association with jets in pp collisions at 8 TeV, JHEP 04 (2017) 022 [arXiv: 1611.03844 ].

[19] The CMS Collaboration, Measurements of the associated production of a $Z$ boson and $b$ jets in pp collisions at 8 TeV, Eur. Phys. J. C 77 (2017) 751 [arXiv:1611.06507].

[20] The CMS Collaboration, Measurement of associated Z + charm production in pp collisions at $8 \mathrm{TeV}$, CMS-PAS-SMP-15-009, https://cds.cern.ch/record/2202823 [arXiv: 1711.02143 ].

[21] The CMS Collaboration, Measurement of electroweak production of a $W$ boson and two forward jets in proton-proton collisions at 8 TeV, JHEP 11 (2016) 147 [arXiv: 1607 . 06975].

[22] The CMS Collaboration, Electroweak production of two jets in association with a $Z$ boson in proton-proton collisions at $8 \mathrm{TeV}$, CMS-PAS-SMP-16-018, https://cds.cern.ch/record/2261499 [arXiv:1712.09814]. 